\title{
Matched Field Processing for complex Earth structure
}

Sven Schippkus and Céline Hadziioannou

Institute for Geophysics, University of Hamburg, Germany, Email: sven.schippkus@uni-hamburg.de

This manuscript is a non-peer reviewed pre-print and has been submitted for publication in Geophysical Journal International. Subsequent versions of this manuscript may have slightly different content.

Please feel free to contact any of the authors; we welcome feedback. 


\title{
Matched Field Processing for complex Earth structure
}

\author{
Sven Schippkus ${ }^{1}$ and Céline Hadziioannou ${ }^{1}$ \\ ${ }^{1}$ Institute for Geophysics, University of Hamburg, Germany, Email: sven.schippkus@uni-hamburg.de
}

\begin{abstract}
Matched Field Processing (MFP) is a technique to locate the source of a recorded wave field. It is the generalization of beamforming, allowing for curved wavefronts. In the standard approach to MFP, simple analytical Green's functions are used as synthetic wave fields that the recorded wave fields are matched against. We introduce an advancement of MFP by utilizing Green's functions computed numerically for real Earth structure as synthetic wave fields. This allows in principle to incorporate the full complexity of elastic wave propagation, and through that provide more precise estimates of the recorded wave field's origin. This approach also further emphasizes the deep connection between MFP and the recently introduced interferometry-based source localisation strategy for the ambient seismic field. We explore this connection further by demonstrating that both approaches are based on the same idea: both are measuring the (mis-)match of correlation wave fields. To demonstrate the applicability and potential of our approach, we present two real data examples, one for an earthquake in Southern California, and one for secondary microseism activity in the Northeastern Atlantic and Mediterranean Sea. Tutorial code is provided to make MFP more approachable for the broader seismological community.
\end{abstract}

\section{Keywords}

Seismic noise, Seismic Interferometry, Interferometry, Wave propagation

\section{Introduction}

The ambient seismic field has become an attractive target of seismological studies over the last two decades (Nakata et al., 2019). Interferometry of this complex wave field, combined with increased 
non-peer reviewed preprint submitted to EarthArXiv

station density, has enabled detailed studies of Earth's structure (e.g., Shapiro et al., 2005; Lu et al., 2018; Schippkus et al., 2018) and its temporal changes (e.g., Brenguier et al., 2008; Hadziioannou et al., 2011). Such studies rely most commonly on seismic wave fields generated by the interaction between the oceans and the solid Earth, so-called microseisms. Understanding the exact mechanism for this interaction has been a challenge for more than half a century (Longuet-Higgings, 1950; Hasselmann, 1963; Ardhuin et al., 2015) and some open questions remain, e.g., about the emergence of Love waves in the secondary microseism (Ziane \& Hadziioannou, 2019; Gualtieri et al., 2020). More recently, other sources such as trains (Fuchs et al., 2017; Brenguier et al., 2019; Liu et al., 2021), wind turbines (Stammler \& Ceranna, 2016; Hu et al., 2019), direct wind-land interaction (Johnson et al., 2019), rain (Dean, 2019), and rivers (Burtin et al., 2008; Smith \& Tape, 2019) have become the focus of several studies investigating high-frequency seismic noise.

To study all of these sources in detail and understand their mechanisms, precise knowledge of their locations is necessary. Dense installations of seismic stations near known sources can provide intriguing insight into the sources' interactions with the solid Earth (Riahi \& Gerstoft, 2015), and can give evidence for previously unrecorded interactions (Schippkus et al., 2020). Installations like these are not widely available, though. For other sources, it may not be technically feasible to install stations close to all expected source locations, e.g., in the deep oceans to study ocean microseisms or in the Earth's subsurface. Beyond the interest in the fundamental principles of seismic wave generation by different sources, studies that rely on interferometry of the ambient seismic field to gain knowledge about Earth's structure ideally incorporate a priori knowledge of source locations to account for the potential bias introduced by their spatial distribution (Fichtner et al., 2017; Sergeant et al., 2020). Strategies of earthquake seismology to locate seismic sources, such as travel-time inversion, are not applicable to ambient seismic noise due to the complexity of the analysed wave field. There is not one single dominant source (e.g., an earthquake or explosion) that results in clearly identifiable and thus exploitable phase arrivals in seismograms across several stations.

Instead, strategies have emerged that aim to quantify the angle of arrival of seismic energy in recorded seismograms, emitted by sources of unknown type (Fig. 1a,b). Polarization analysis exploits the particle motion of the seismic wave field at one location $\vec{x}_{j}$, resolved by three-component seismometers (Fig. 1a, e.g., Schimmel \& Gallart, 2003). Depending on the analysed wave type, the particle motion gives an indication of the angle of arrival. When combining results from multiple stations, this analysis can be used to triangulate the source location $\vec{x}_{s}$ (e.g., Schimmel et al., 2011). However, a number of assumption have to be made, e.g., great-circle propagation, as well as proper 
non-peer reviewed preprint submitted to EarthArXiv

identification and clear separation of wave types. This approach can be a first step in understanding the recorded wave field, but is often quite tricky in practice, especially on recordings of ambient seismic noise (Gal \& Reading, 2019).

Beamforming is a source localisation approach based on the assumption that seismic waves propagating across seismic arrays can be treated as plane waves, if their wavelengths are much larger than the aperture of the array (Fig. 1b). To test whether a candidate plane wave - characterised by its horizontal slowness or equivalently arrival angle and apparent velocity - was recorded on the array, expected relative time delays $\Delta t\left(\vec{x}_{j}, \vec{x}_{k}\right)$ between the stations are computed and corrected for. This is called delay-and-sum beamforming, where each seismogram is shifted in time and summed together, forming the beam (Rost \& Thomas, 2002). The quality of the beam is evaluated, giving the so-called beampower. Other formulations of this method exist, e.g., an equivalent cross-correlation approach (Ruigrok et al., 2017). Beamforming has been widely adopted by the seismological community and is currently the standard tool for identifying sources of the ambient seismic field (Gal \& Reading, 2019, and references therein). Recent advances focus on incorporating three-component seismograms (Riahi et al., 2013; Juretzek \& Hadziioannou, 2016, 2017), avoiding bias introduced by averaging across broad frequency bands (Gal et al., 2014), or estimating surface wave anisotropy directly from beamforming (Löer et al., 2018). Beamforming has its main advantages in computational speed, little if any data processing, and high time resolution. Its main drawbacks all result from the planewave assumption: sources have to be far from the array, the wave field has to be strictly coherent across stations, and the array geometry limits the resolution capabilities (Rost \& Thomas, 2002). For a recent review of beamforming and polarization analysis see Gal \& Reading (2019).

A new source localisation strategy based on seismic interferometry has been introduced in recent years as an attractive alternative, sometimes referred to as kernel-based source inversion (Ermert et al., 2016). The goal of this approach is not to determine the angle of arrival, but to directly quantify the distribution of seismic sources in space. Interferometry of the ambient seismic field recorded on multiple stations gives new wave fields, propagating to and from the respective reference stations (Fig. 1c, Aki, 1957; Wapenaar et al., 2010; Campillo \& Roux, 2015; Fichtner et al., 2017). An inhomogeneous distribution of sources results in asymmetric cross-correlation functions, indicated by the thickness of the wave fronts in Fig. 1c (Paul et al., 2005). In practise, this asymmetry is usually quantified by comparing the causal and acausal part of each correlation function. In the interferometry-based approach, synthetic cross-correlation functions are computed for a given source distribution and compared against cross-correlation functions from real data. The mismatch between 
non-peer reviewed preprint submitted to EarthArXiv

the two is evaluated (e.g., by quantifying amplitude asymmetry), the source model perturbed, and a best-fit source distribution is found via gradient descent in an iterative manner (Ermert et al., 2016). Recent work has focused on improving efficiency (Igel et al., 2021b), the mismatch measure (Sager et al., 2018), or expanding the method to multiple frequencies (Ermert et al., 2021). The advantages of this approach are the stability of results, not as strict requirements on station geometry, and a comprehensive theoretical foundation. Its disadvantages lie in computational cost, treatment of recorded data, through that introduction of assumptions, and loss of temporal resolution.

Another approach that has gained some popularity in seismology in recent years is Matched Field Processing (MFP). MFP is the generalisation of beamforming to allow arbitrary wavefronts (Baggeroer et al., 1993). This approach has been developed in ocean acoustics, where coherency of the wave field emitted by transient sources is high even for stations far away. Candidate sources are defined in space and absolute travel times $t\left(\vec{x}_{j}, \vec{x}_{s}\right)$ are computed based on true distance to the source (Fig. 1d). Synthetic wave fields are computed for these travel times and matched against the recorded wave field. In the seismological context, MFP has been applied succesfully on local (Corciulo et al., 2012; Umlauft \& Korn, 2019; Umlauft et al., 2021) and regional scale (Gal et al., 2018). Recent developments in MFP include the development of different beamformers (e.g., Zhu et al., 2020), improved estimation of travel times (Gal et al., 2018), or estimating synthetic wave fields empirically (Gibbons et al., 2017). MFP is an attractive strategy for source localisation of the ambient seismic field. It allows for curved wavefronts, is based on only few assumptions, requires no intermediate step such as pre-processing of recordings, and retains computational efficiency. While the plane-wave assumption is neglected in MFP, coherency of the wave field across stations is still required for good results. This poses challenges when analysing recordings for stations that are not close together, and especially so for ambient seismic noise.

In this paper, we introduce an advancement of MFP to incorporate real Earth structure and account for the complexity of seismic wave propagation. In the following, we introduce the standard MFP approach, demonstrate its shortcomings, and present our solution by incorporating realistic Green's functions. We discuss implications of our approach, strategies to cope with some of them, how different disciplines and localisation approaches intersect, and finally demonstrate the applicability of our approach on two real data examples. 


\section{Matched Field Processing}

The MFP algorithm is straight-forward: For a given potential source location, a synthetic wave field is computed and matched against the recorded wave field, i.e., the seismograms, taking coherency of the wave fields across stations into account. This match is evaluated and compared against other potential source locations. The potential source location with the highest score or beampower (representing the best-matching synthetic wave field) is the resolved source location.

More precisely, spectra $d\left(\omega, \vec{x}_{j}\right)$ are computed from the recorded seismograms at each receiver position $\vec{x}_{j}$. The cross-spectral density matrix is computed as

$$
K_{j k}(\omega)=d^{*}\left(\omega, \vec{x}_{j}\right) d\left(\omega, \vec{x}_{k}\right)
$$

with $*$ denoting the complex conjugate. $K_{j k}(\omega)$ holds all information about the recorded wave field and encodes its coherency across stations; it contains the cross correlations of the seismograms from all station pairs. Following Bucker (1976), auto correlations are excluded, i.e., only components $k \neq j$ are computed and later utilized. This is particularly useful in the context of ambient seismic noise, because noise wave fields are likely to be only weakly coherent across stations.

The synthetic wave field, i.e., the seismograms expected at each station from the candidate source, is represented through synthetic spectra $s\left(\omega, \vec{x}_{j}, \vec{x}_{s}\right)$, with $\vec{x}_{s}$ the source position and $\vec{x}_{j}$ the receiver position. In principle, these could be estimated in the time domain, but MFP computations are done in frequency domain for simplicity and computational speed. More on how these are computed in practise in section 2.1 and onwards.

The match of the two wave fields represented through $K_{j k}(\omega)$ and $s\left(\omega, \vec{x}_{j}, \vec{x}_{s}\right)$ is then estimated through a so-called beamformer or processor. The most straight-forward beamformer is the conventional beamformer, which in its most compact form in vector notation is often written as (e.g., equation 25 in Baggeroer et al., 1993)

$$
B=\mathbf{s}^{*} \cdot \mathbf{K} \cdot \mathbf{s},
$$

with $B$ the beampower score for a potential source location. In literature, this beamformer is sometimes called Bartlett processor, although the origin of this name is unclear (e.g., Gal \& Reading, 2019), linear beamformer (e.g., Baggeroer et al., 1993), or frequency-domain beamformer (DeMuth, 1977). We express $B$ more explicitly, excluding auto correlations, for clarity as

$$
B=\sum_{\omega} \sum_{j} \sum_{k \neq j} s_{j}^{*}\left(\omega, \vec{x}_{j}, \vec{x}_{s}\right) K_{j k}(\omega) s_{k}\left(\omega, \vec{x}_{k}, \vec{x}_{s}\right) .
$$


non-peer reviewed preprint submitted to EarthArXiv

Other estimators of beampower exist, and their development is an active field of research (e.g., Capon, 1969; Schmidt, 1986; Cox et al., 1987; Cox, 2000; Gal et al., 2014; Zhu et al., 2020). Beamformers are often classified into conventional (eq. 3), adaptive (e.g., Capon, 1969; Cox et al., 1987; Cox, 2000) and sub-space beamformers (e.g., Schmidt, 1986). Adaptive beamformers aim to increase resolution of the beampower distribution by increasing sensitivity to signal, but inherently rely on high signal-to-noise ratio (SNR). The increased resolution is also accompanied by increased computational cost, e.g., the Capon beamformer involves computing the inverse of $K_{j k}(\omega)$ (Capon, 1969). Sub-space detectors such as MUSIC (Schmidt, 1986) involve computation of the eigenvectors of $K_{j k}(\omega)$, and making a selection of those for further computations based on which eigenvectors contribute to the signal and which are "noise". Corciulo et al. (2012) used a similar approach and were able to resolve multiple sources this way. One of the expressed goals of the approach we introduce in this paper is to be able to locate sources of ambient seismic noise, and as such SNR is by defintion low. Beamformers beyond the conventional beamformer may not be appropriate for this, because they either require high SNR or a choice of what part of the cross-spectral density matrix is signal and what is "noise". Krim \& Viberg (1996) have addressed the question of which beamformer performs best under what circumstances for standard MFP before. A detailed analysis of this in the context of our approach we introduce here is beyond the scope of this paper.

\subsection{Synthetic wave field in the standard approach}

In practise, assumptions and simplifications about structure and wave propagation have to be made in order to compute the synthetic wave fields $s\left(\omega, \vec{x}_{j}, \vec{x}_{s}\right)$ that the recorded data are matched against. In most seismological and almost all ocean acoustics applications so far, simple analytical Green's functions of the form

$$
s\left(\omega, \vec{x}_{j}, \vec{x}_{s}\right)=e^{-i \omega t\left(\vec{x}_{j}, \vec{x}_{s}\right)},
$$

are used, with $t\left(\vec{x}_{j}, \vec{x}_{s}\right)$ the travel time of the investigated wave between source and receiver (Fig. 1d). In some seismological studies, the addition of an amplitude term $A\left(\vec{x}_{j}, \vec{x}_{s}\right)$ that accounts for geometrical spreading and/or inelastic attenuation has been discussed (Corciulo et al., 2012; Bowden et al., 2020). The goal of such a term would be to increase the accuracy of the synthetic wave field by incorporating some of the seismic waves' propagation behaviour. Neglecting the amplitude term entirely, as is usually done, makes standard MFP equivalent to delay-and-sum beamforming without the plane-wave assumption (Bucker, 1976). More on this in section 2.4.

In the simplest possible study target, i.e., a single stationary source in an isotropic, homogeneous 
non-peer reviewed preprint submitted to EarthArXiv

medium with constant velocity $v=$ const and only straight-ray propagation of a single phase, the travel time is simply $t\left(\vec{x}_{j}, \vec{x}_{s}\right)=\Delta x / v$. This requires prior knowledge of $v$ and the assumption that $v=$ const is a good approximation of the medium. In seismology, this approach has been successfully demonstrated on local scale (e.g., Corciulo et al., 2012; Umlauft \& Korn, 2019; Umlauft et al., 2021), where propagation effects due to heterogeneous Earth structure can be neglected. Without any prior knowledge of the velocity structure, another approach is to treat $v$ as an additional dimension in the parameter space that needs to be explored, though this can become computationally quite expensive and may require sampling strategies other than a standard grid search (Gradon et al., 2019).

On regional scale, Gal et al. (2018) estimated $t\left(\vec{x}_{r}, \vec{x}_{s}\right)$ from already available phase velocity maps using Fast Marching Method (Sethian, 1999), which accounts for off-straight-ray propagation of surface waves, and by that incorporating some complexity of wave propagation in real Earth structure. This approach also inherently incorporates frequency-dependent effects, i.e., $t\left(\vec{x}_{r}, \vec{x}_{s}\right)$ becomes $t\left(\vec{x}_{r}, \vec{x}_{s}, \omega\right)$. Gal et al. (2018) used this to study the primary ( $16 \mathrm{sec}$. period) and secondary $(\sim 8 \mathrm{sec}$. period) microseism separately by estimating phase travel times from their respective phase velocity maps. This approach requires knowledge of the analysed wave type. Gal et al. (2018) solve this by rotating seismograms into the radial/transverse-system and assuming that for the chosen frequencies (microseism), surface waves are dominant and only recorded on their respective component (Love on transverse, Rayleigh on radial). This is a reasonable assumption, commonly made when analysing ocean microseism (Nakata et al., 2019), but may not always be appropriate depending on the study target. Incorporating multiple phases (e.g., a mix of body and surface waves) at the same frequency is not straight-forward with the standard approach and clearly requires further assumptions about the number of phases and their respective travel times, increasing the parameter space considerably. Furthermore, when investigating frequencies at which the identification of wave types may be challenging, this strategy potentially misses or misattributes important information in the recorded wave field and may bias results. Approaching the complexity of wave propagation in real Earth structure in this manner seems quite cumbersome and impractical, as it ends up requiring manual consideration of all of these effects.

\subsection{Numerical synthetic wave fields for complex Earth structure}

We propose to use Green's functions computed numerically for Earth structure directly as the synthetic wave field $s\left(\omega, \vec{x}_{r}, \vec{x}_{s}\right)$ ("our approach") instead of the analytical form described above (eq. 4, "standard approach"). Effects such as dispersion and multiple wave types are then inherently ac- 
non-peer reviewed preprint submitted to EarthArXiv

counted for, even for simple 1D media. If the Green's function are computed for a 3D Earth, further effects such as focusing and defocusing, wave-type conversion, and coupling can all be accounted for, increasing the precision of this approach further.

We demonstrate our method with synthetic examples for a broadband and a narrowband explosion source (Fig. 2). The setup consists of two small arrays of three stations each that record the wave field emitted by a seismic source located at the surface between them. The medium is a 3D axisymmetric Earth (Nissen-Meyer et al., 2014), based on PREM (Dziewonski \& Anderson, 1981). The "recorded" seismograms are computed for the same model and incoherent noise is added. With the standard MFP approach (assuming $t\left(\vec{x}_{r}, \vec{x}_{s}\right)=\Delta x / v$ ), locating the source precisely is quite challenging for both broad- and narrowband sources (Fig. 2a, c). The resolved location is clearly sensitive to the chosen velocity of the medium $v$. When the chosen velocity is too low, the resolved source lies further away than the real source. When it is too high, the resolved source lies closer. This applies to both broadband and narrowband sources (Fig. 2a, c). For the broadband source, the highest frequency available in the numerical Green's functions is $0.2 \mathrm{~Hz}$. The error in location introduced for $v=3.0 \mathrm{~km} / \mathrm{s}$ is smaller for the broadband source than for the narrowband source. This occurs, because the broadband wave field contains phases that are of different type and travel with different velocities, and $v=3.0 \mathrm{~km} / \mathrm{s}$ is a good estimate for at least some of them. For the narrowband wave field, which contains mainly Rayleigh waves at $0.13-0.15 \mathrm{~Hz}, v=3.0 \mathrm{~km} / \mathrm{s}$ is already clearly too slow. For surface waves in particular, a different choice of velocity $v$ for each analysed frequency band would seem appropriate due to their dispersive nature (Gal et al., 2018).

With Green's functions computed numerically for the same Earth structure, the location of the source is resolved precisely for both broad- and narrowband sources (Fig. 2b, d). This is unsurprising, given that we are essentially matching the synthetic wave field against itself with some noise. But this is also exactly the intent behind the approach: matching the recorded wave field with a more realistic synthetic wave field. Our simple synthetic tests show that the standard approach can be imprecise for locating realistic sources in slightly complex media, even under ideal conditions, and is highly dependent on choosing the correct velocity. With our approach, we do not have to consider frequency-dependant effects explicitly as long as the numerical Green's functions applied are a good representation of elastic wave propagation.

MFP for narrowband sources results in prominent side lobes of beampower, regardless of approach (Fig. 2c,d). These are interference patterns that emerge because MFP is ultimately a correlationbased measure of waveform fit (more on that in section 2.4). The exact shape and position of 
non-peer reviewed preprint submitted to EarthArXiv

sidelobes depends on the station distribution and wavelength of the investigated wave, while the correct location does not. Sidelobes will be suppressed, if a wide frequency band is used (Fig. 2a,b) or several runs of MFP for narrow neighbouring frequency bands are stacked (Umlauft et al., 2021). MFP originated as a narrowband localisation technique (Bucker, 1976) and has been adopted for broadband sources thereafter (e.g., Baggeroer et al., 1993; Brienzo \& Hodgkiss, 1993; Soares \& Jesus, 2003), where the suppression of sidelobes plays a role. This has some implications for the resolution capability of MFP, which depends heavily on whether the analysed source emits a wide frequency band or not. These interference patterns can also be thought of as a trade-off between spatial and frequency resolution of MFP. Using more precise Green's functions has in principle no impact on this.

The basic idea of incorporating more realistic Green's functions in MFP is not new. In ocean acoustics, waveforms are coherent across large distances due wave propagation being focused in the SOFAR channel, but MFP results can be highly sensitive to acoustic wave velocities (Tolstoy, 1989), similar to what we have shown in Figure 2. Bathymetry and multiple reflections may complicate the recorded wave field even further and impact MFP performance significantly, and thus should ideally be incorporated (e.g., D'Spain et al., 1999). For elastic waves in solid Earth structure, further effects would need to be considered, as described above. One approach to this is empirical Matched Field Processing (Gibbons et al., 2017). Gibbons et al. (2017) estimate empirical Green's functions for each station from recordings of known sources by computing the principal eigenvector of the covariance matrix of the incoming wave field for two nearly identical sources. They have demonstrated their approach in the context of mining blasts. The obvious limitation is that such template sources are required, which allows its application only for certain scenarios.

Our approach does not have this limitation. Using numerically computed synthetic wave fields, we can place candidate sources wherever we want. Our approach is then mainly limited by the accuracy of the numerical model and computation strategy. Improving MFP in this way has only become possible recently thanks to efforts by other authors to improve the computation of databases of Green's functions for real Earth structure and provide them to the community (e.g., Nissen-Meyer et al., 2014; van Driel et al., 2015; Krischer et al., 2017; Heimann et al., 2017). Computing Green's functions for complex Earth structure is expensive, which is why we rely in our analysis on pre-computed databases using instaseis (van Driel et al., 2015). Green's functions databases for realistic Earth structure up to frequencies of the secondary microseism are available for download at IRIS-DMC (Hutko et al., 2017) or Pyrocko Green's Mill (Heimann et al., 2017). 
non-peer reviewed preprint submitted to EarthArXiv

\subsection{On amplitudes in MFP}

The standard approach does not include an amplitude term. When it is incorporated, it ideally describes the two contributions for geometrical spreading and inelastic attenuation on the amplitudes (e.g., Bowden et al., 2020). Computing both requires assumptions about wave type and the attenuation properties of the Earth, again increasing the parameter space. Bowden et al. (2020) show in a synthetic example that first applying and later correcting for this amplitude term does not improve source locations compared to neglecting it from the beginning. It merely tests whether the assumed wave type and quality factor are correct, which poses the danger that wrong assumptions may bias results in real data studies, but also opens the opportunity to constrain anelastic properties of the Earth, if the source locations are already well-known. More importantly though, Bowden et al. (2020) also showed that computing MFP results including the amplitude term in the synthetic Green's function without correcting for it is equivalent to mapping out the sensitivity kernel for the given station-source distribution. As the authors have pointed out, MFP and interferometry-based localisation are closely connected (more on this in section 2.4). MFP without correcting for amplitudes is not useful for directly locating sources (as the highest score is no longer necessarily at the source location), but can be an appropriate starting model for the interferometry-based strategy (lgel et al., 2021a).

A strategy similar to the interferometry-based scheme, where the source strength at a position is perturbed and the fit between model and data is evaluated, is not viable for MFP itself. The beampower at a potential source location scales linearly with the absolute amplitudes of the recorded seismograms. This is the case, even if the match in amplitude decreases, because MFP is ultimately summing over correlations of waveforms. For this reason, other measures of waveform-similarity that account for a mismatch in amplitude are commonly applied in other approaches, e.g., in full waveform inversion (Yong et al., 2019, and references therein). Accounting for this behaviour directly in MFP is currently not possible, because it is inherent to how beamformers are designed.

Therefore, a strategy is required to correct for amplitude terms. Numerically computed Green's functions for real Earth structure inevitably contain amplitude terms. Several approaches may appear reasonable to correct for them: correcting for amplitude decay (Fig. 3b), time-domain normalisation (Fig. 3c), and spectral whitening (Fig. 3d). Without any treatment of amplitudes, the beampower distribution is heavily biased by distance to stations (Fig. 3a). This effect is more pronounced compared to Bowden et al. (2020), because our Green's functions also contain body waves. Only a zoomed-in view allows to see the distribution of beampowers with a linear colorscale. The retrieved 
non-peer reviewed preprint submitted to EarthArXiv

source location without amplitude treatment is close to one of the stations nearest to the actual source at the center.

Applying a correction factor for geometrical spreading of surface waves as has been demonstrated by Corciulo et al. (2012) corrects for some but not all of the amplitude bias (Fig. 3b). The beampower peak is still found near a station, because body waves are not corrected for. It is not clear how a single correction term could be designed to correct for both body and surface waves simultaneously. When we neglect the near-station beampowers, we find a local maximum (small red circle) near the correct source location. We are not able to resolve the source location correctly. For now, we advise against application of a correction term for amplitude decay, because it requires assumptions about wave type and the medium's inelastic properties, opening up room for error and bias as demonstrated here. When synthetic Green's function contain only a single wave type, applying a correction term is a viable strategy as shown by Bowden et al. (2020). In real applications and without prior knowledge of the source location (which defeats the purpose of MFP), such bias seems not trivial to resolve. More drastic approaches to dealing with amplitude-induced bias are necessary.

Time-domain normalisation aims to completely remove the impact of amplitudes by converting the synthetic wave fields to time domain $s\left(t, \vec{x}_{j}, \vec{x}_{s}\right)$ and dividing those by their maximum amplitude. With this approach, we resolve the beampower peak close to the true source (Fig. 3c), but introduce ripple-shaped artefacts in the entire beampower distribution. Time-domain normalisation is only then equivalent to properly removing the effect of amplitude decay, if waveforms did not change their shape across stations. Elastic wave propagation in realistic Earth structure results in the emergence of different phases depending on source-receiver distance, changes to the waveforms due to dispersion, as well as their amplitudes being affected differently by decay. These effects introduce the observed pattern, which is undesirable.

Spectral whitening or frequency-domain normalisation is the process of dividing the frequency spectrum by its amplitude spectrum, a technique commonly applied in processing of ambient seismic noise records for interferometry (Bensen et al., 2007; Fichtner et al., 2020). Neglecting amplitudes as done in the standard approach is equivalent to whitening of synthetic Green's functions. In fact, whitening of the synthetic wave field is applied in early formulations of standard MFP (equation 24 in Baggeroer et al., 1993). In the context of interferometry of the ambient seismic field, whitening is often performed with a water-level or smoothed amplitude spectrum to stabilise the procedure numerically and not over-emphasize frequencies that carry no useful information (Bensen et al., 
non-peer reviewed preprint submitted to EarthArXiv

2007). Because we treat the synthetic spectra only, we are not concerned with smoothing of the amplitude spectrum before division and artefacts that whitening may introduce in real data and directly perform whitening as

$$
s_{\text {white }}\left(t, \vec{x}_{j}, \vec{x}_{s}\right)=\frac{s\left(t, \vec{x}_{j}, \vec{x}_{s}\right)}{\left|s\left(t, \vec{x}_{j}, \vec{x}_{s}\right)\right|} .
$$

This approach successfully retrieves the correct source location and does not appear to introduce any unwanted biases (Fig. 3d).

From our tests, whitening the spectra of the synthetic wave fields (Fig. 3d) appears to be the most advantageous approach, and follows the original formulation of MFP (Baggeroer et al., 1993). It introduces no alteration of the recorded data, eliminates attenuation and spreading effects, removes potential issues caused by source strength, and successfully retrieves the true source location. With this approach, all sources are weighted equally likely, regardless of distance to the receivers, as long as their wave fields are well-recorded on all stations. This may not always be an advantage, e.g., in global-scale studies, where the convergence of the wave field at the source's antipode may introduce bias. By whitening we also lose the ability to, in principle, constrain anelastic parameters of the Earth, but it is not clear to us how that could be approached for numerically computed Green's functions that contain all wave propagation effects.

\subsection{Naming conventions and conceptual approaches to MFP}

To illustrate how literature from multiple discplines intersects, we want to take a moment to clarify different naming conventions and how MFP can be understood conceptually in different ways.

In this paper, we have used language that describes the results of MFP as the distribution of beampower retrieved from matching recorded wave fields with synthetic wave fields or Green's functions $s\left(\omega, \vec{x}_{j}, \vec{x}_{s}\right)$ for candidate source locations. This language, particularly Green's functions, is natural for seismologists (e.g., Gibbons et al., 2017; Umlauft \& Korn, 2019), though rarely also used in ocean acoustics studies (e.g., Li et al., 2021).

In ocean acoustics, other terminology is more common for some of these concepts. The distribution of beampowers may be called ambiguity surface (Bucker, 1976), intended to express the emergence of sidelobes for narrowband sources (Fig. 2c,d). $s\left(\omega, \vec{x}_{j}, \vec{x}_{s}\right)$ is sometimes called steering vector, expressing the idea that the array is "steered" towards the source during beamforming or MFP, or replica vector, communicating that the vector represents a replica of the expected wave field (Baggeroer et al., 1993). 
non-peer reviewed preprint submitted to EarthArXiv

Still, both the seismological and ocean acoustics communities understand MFP as matching of wave fields; this idea is the original concept introduced by Bucker (1976), and gives an intuitive understanding of the physics involved. Above, we mentioned that array beamforming for plane waves is a special case of MFP. For plane-wave beamforming, Green's functions of the form $s\left(\omega, \vec{x}_{j}, \vec{x}_{s}\right)=$ $e^{-i \omega t\left(\vec{x}_{j}, \vec{x}_{s}\right)}$ are used, and only the manner in which $t\left(\vec{x}_{j}, \vec{x}_{s}\right)$ is estimated is adapated to use relative distances perpendicular to the plane wavefront (Fig. 1b) instead of distances to potential source locations (Fig. 1d). In that case, MFP is exactly delay-and-sum beamforming, regardless of whether plane waves or curved wavefronts are used (Bucker, 1976). In this formulation (what we called the "standard approach" to MFP) the Green's function can be understood in two ways: It is the wave field emmitted by a point source (the impulse response), if the medium is an isotropic, homogeneous half-space. It also represents a phase shift (or time-delay), if it is convolved with a waveform.

Understanding beamforming as convolution leads to another way of conceptualising MFP. We rewrite equation 3 , omitting the variables $\left(\omega, \vec{x}_{j}, \vec{x}_{s}\right)$ for readability, as

$$
B=\sum_{\omega} \sum_{j} \sum_{k \neq j} s_{j}^{*} d_{k}^{*} d_{j} s_{k}=\sum_{\omega} \sum_{j} \sum_{k \neq j}\left(s_{j}^{*} s_{k}\right)\left(d_{k}^{*} d_{j}\right) .
$$

Here, $d_{k}^{*} d_{j}$ is the correlation of the recorded wave fields and $s_{j}^{*} s_{k}$ correlation of the synthetic wave fields for each station pair $k, j$. The "matching" of wave fields in MFP is exactly this: convolution of their correlation wave fields.

This is particulary relevant, because it further demonstrates the close connection between MFP and the interferometry-based localisation strategy, and gives a different perspective to the insights provided by Bowden et al. (2020). In both approaches, cross-correlation functions of recorded data and of synthetic data are computed and compared against each other. The main difference between them lies in how exactly cross-correlation functions are computed and how the (mis-)fit between the two is evaluated. It is then not surprising that MFP results are a good starting model for interferometry-based localisation (Igel et al., 2021a); in a very real sense MFP is interferometrybased localisation, just with less processing and a different mismatch measure. Bowden et al. (2020) have described this connection more mathematically: starting from cross-correlation beamforming (Ruigrok et al., 2017), a simple change in the order of operations - from shifting waveforms first and then computing the cross-correlation coefficient to first computing the correlation function and then measuring at the corresponding time lag - creates an equivalency (under certain conditions) between MFP and interferometry-based source inversion. This description and the one we introduce above result in the same realisation: fundamentally, there currently exists only one approach for locating 
non-peer reviewed preprint submitted to EarthArXiv

sources of ambient seismic noise in the sense that the coherency of the recorded wave field across stations is the most important aspect that enables source localisation. That both approaches are fundamentally the same may not be intuitive at first, especially considering the strikingly different sketches to illustrate them (Fig. 1c,d), and the different language both communities use.

To retrieve " reliable" cross-correlation functions of the recorded data in ambient noise seismology, processing and stacking over time is common (Fichtner et al., 2020). Such cross-correlation functions are often called estimated or empirical Green's functions, relying on assumptions of homogeneous noise source distribution or wave field equipartition (e.g., Nakata et al., 2019). These are not to be confused with what Gibbons et al. (2017) called empirical Green's functions. Note that Fichtner et al. (2017); Ermert et al. (2016) and others purposefully avoid naming and understanding crosscorrelation functions as empirical Green's functions and do not rely on the above assumptions. The fact that these assumptions are incorrect is in fact what enables the interferometry-based strategy.

MFP is similarly not concerned with such assumptions about the equipartion or source distribution of the recorded wave field. Furthermore, it foregoes processing of seismograms for stability entirely, allowing for high time-resolution and avoiding artefacts potentially introduced by the processing (Fichtner et al., 2020). Importantly though, the mismatch measure employed in MFP does not allow iterative inversion by source-strength perturbation, because convolution (or correlation) does not account for amplitude mismatch. If signals are in phase, increasing amplitudes of one results in linearly-scaling beampowers regardless of how well the waveforms fit. At this point, it is clear that both communities may benefit from each other, as has already rightfully been pointed out by Bowden et al. (2020). It is in principle possible to employ strategies of the ambient seismic noise community to "improve" the correlation functions $d_{k}^{*} d_{j}$. A detailed analysis of the advantages and disadvantages this would bring, and what exactly "improving" would mean in the context of MFP is beyond the scope of this paper. Similarly, increasing the accuracy of MFP in a seismological context and discussing its fundamental ideas and limitations, as is the intent of this paper, may benefit developments in the larger field of ambient seismic noise localisation.

\subsection{Limitations of MFP}

Above, we have already explored the advantages and limitations of using numerically computed synthetic wave fields (Fig. 2) and amplitudes (Fig. 3) in MFP, as well as the emergence of striped interference patterns for narrowband sources (Fig. 2). MFP shows further undesired behaviour under certain conditions that we encounter in real-world applications. Some of these are more 
non-peer reviewed preprint submitted to EarthArXiv

straight-forward to understand in the conceptual framework of convolution introduced above.

\subsubsection{Source-Station Geometry}

For beamforming, the impact of an array's geometry on its resolution capability is well studied, and quantified by the array-response function (Rost \& Thomas, 2002). Standard MFP becomes planewave beamforming for very large distances between source and array, because accounting for curved wavefronts has negligible impact on travel times. In that case, the lessons learned in beamforming, e.g., what wavelengths are resolvable without aliasing, apply one-to-one. When MFP is considered as an approach, the source-station geometry should be such that accounting for curved wavefronts actually has useful impact on the results, i.e., the difference in expected travel times compared to plane waves is much larger than the expected measurement error. Because MFP is not bound to the plane-wave assumption, there is no meaningful difference between treating a collection of stations as an array or a network. Still, the inter-station distance should not be much smaller than the investigated wavelength or incoherent noise may prevent being able to reliably resolve the source location. In MFP, quantifying resolution in a similar manner to the array-response function for beamforming is an unsolved problem, because the parameter space is much larger.

Closely related to these considerations is that high waveform coherency is required across stations, regardless of approach. In standard MFP or beamforming, i.e., $s\left(\omega, \vec{x}_{j}, \vec{x}_{s}\right)=e^{-i \omega t\left(\vec{x}_{j}, \vec{x}_{s}\right)}$, coherency means retaining the exact shape of the waveforms across stations, because waveforms are simply shifted in time. In our approach, waveform coherency takes a slightly different meaning, because elastic wave propagation can change the shape of recorded waveforms significantly. So instead, waveforms need to be coherent after elastic wave propagation effects have been accounted for, in our approach via synthetic Green's functions for real Earth structure. This is an important distinction, as our approach losens the requirements on station geometry for MFP quite drastically. In principle, stations on opposite sides of the Earth can be used simultaneously for MFP, as long as the wave propagation effects are properly incorporated and the signal is recorded above the noise-level on both stations. Therefore, our approach improves upon this limitation of MFP, and we make use of this in the real data examples below.

\subsubsection{Station Density}

Station density has direct impact on the retrieved beampower distribution that is worth pointing out explicitly (Fig. 4a). In a synthetic test, we place four times the stations, with the same coordinates, 
non-peer reviewed preprint submitted to EarthArXiv

on the right side than we do on the left (Fig. 4b). The beampower distribution shows a visual bias towards the top-left, caused simply by the presence of more stations that recorded the signal in the bottom-right. While in the ideal scenario here, the exact source location is still resolved correctly, interpreting this distribution without prior knowledge of the sources in a real-world application seems challenging. This bias in MFP results follows directly from understanding MFP as the sum over convolutions of correlated wave fields, as described above. Regions with higher station density are then inherently weighted higher and cause the observed effect.

This goes beyond increased resolution due to better suppression of incoherent noises, and is an effect that essentially all real-world applications of MFP will have to take into consideration. We have tested two possible approaches to correct for this without success. Introducing a coherency-weight where stations that recorded similar waveforms are down-weighted to counter-act the described behaviour, does not improve the retrieved beampower distribution. This approach further loses the advantages that multiple measurements at similar positions can reduce impact of incoherent noise. A different approach may be to homogenise the station distribution, but this often excludes highquality stations from the analysis, especially for permanent arrays. The loss in quality recordings does not seem to be desirable here.

\subsubsection{Multiple Sources}

Single sources can cause prominent interference patterns for narrowband sources (Fig. 2c,d), which depend on station geometry and frequency band. This leads to even more complex, secondary interference when multiple sources are active at the same time. In a synthetic test, we place two narrowband sources that excite identical wave fields simultaneously (Fig. 4b). The second source is placed such that it lies at the edge of a sidelobe of the first source (Fig. 2d). From the retrieved distribution of beampowers it is not at all obvious that two and only two sources are active here, and instead this may be misidentified as a single source close to the left array (Fig. 4b). The new beampower peak is entirely an interference artefact. This smearing of resolved source locations clearly relates to the wavelength of the investigated waves, and similar issues are well-known in the beamforming community, as described above. When the two sources placed are broadband instead (Fig. 4c), one may interpret the beampower distribution as two sources. The true locations are not recovered, with a smaller error for the closer source. Similar problems, such as smeared beampower distributions can occur for single sources that move during the investigated time frame (Li et al., 2021). 
non-peer reviewed preprint submitted to EarthArXiv

These effects briefly demonstrate the, in our view, most important limitation of MFP: the concrete interpretation of individual MFP results. Interpretation seems quite challenging when either stations are distributed heterogeneously or multiple sources are nearby and may have interfering sidelobe patterns. Both conditions are true for most real-world applications. This is one of the main reasons other beamformers are being developed (e.g., Capon, 1969; Schmidt, 1986; Cox et al., 1987; Cox, 2000; Gal et al., 2014; Zhu et al., 2020). In future work, exploring their applicability to and further developing them in the context of elastic waves propagating in real Earth structure, seems like a clear way forward. A convenient way of quantifying MFP's resolution would be exceptionally useful, but is not known to us. Here the interferometry-based localisation strategy shows its strength, with a clear strategy for iteratively getting better estimates of source location.

\subsubsection{Time window length}

In MFP, a choice has to be made on how long of a time window is analysed. The basic requirement is that the time needs to be long enough to record the correlated wave field propagating across all stations, which can be estimated roughly from expected wave velocities. Because MFP is based on correlation wavefields, by default the entirety of the chosen time window influences the result. This is easier to understand with the delay-and-sum concept, where waveforms are shifted in time and summed. Because the entire waveforms are used to compute the sum, all of the waveform plays a role. This limits the time resolution of MFP and has implications depending on the type of source one aims to investigate. If a source is exciting energy repeatedly, the wave field contains more and more of that source's energy the longer the time window is and thus gets weighted higher and higher. This is very useful for stationary "noise" sources. For impulsive sources that act rarely, this can be a disadvantage and time windows should be chosen as small as possible for them. To address this issue, the concept of a windowing function as developed for the interferometry-based localisation strategy (Bowden et al., 2020), may be an opportunity to increase MFP's time resolution even further in the future.

\section{Demonstration on real data}

We demonstrate our approach on two real data examples. 


\subsection{Chino Hills Earthquake}

First, we benchmark our approach with an earthquake in Southern California, the $M_{W}=5.4$ Chino Hills earthquake of 2008-07-29 (Fig. 5). When applying the standard MFP approach, with an assumed velocity $v=3.2 \mathrm{~km} / \mathrm{s}$ (the best fit in the synthetic test in Fig. 2), we find a relatively good location of the earthquake with $7.7 \mathrm{~km}$ distance to the location in the $\mathrm{Cl}$ catalog (Fig. 5a, SCEDC, 2013). The good fit here confirms what other authors have found before: standard MFP can already perform quite well in seismological studies (Gal et al., 2018; Umlauft \& Korn, 2019; Umlauft et al., 2021). When we replace $s\left(\omega, \vec{x}_{j}, \vec{x}_{s}\right)$ with numerical Green's functions (our approach) for an explosive source mechanism, we at first find a decrease in location accuracy (Fig. 5b). The retrieved location is $18.3 \mathrm{~km}$ away from the $\mathrm{Cl}$ location. When we incorporate the moment tensor solution from the $\mathrm{Cl}$ catalog (SCEDC, 2013), trivial to do with our approach, we find an improvement in location accuracy with a distance of only $1.9 \mathrm{~km}$ to the $\mathrm{Cl}$ location (Fig. 5c). This demonstrates one of the potential use cases for MFP with numerical Green's functions: Searching for the best-fitting moment tensor may help constrain the source mechanism of unknown weak sources. A related strategy has been employed by Umlauft et al. (2021). The authors flipped the sign of waveforms, based on visual inspection and expert judgement, before applying MFP. The spatial distribution of whether a waveform had to be flipped or not to increase waveform-coherency across stations, gives hints on the radiation pattern and thus source mechanism of the seismic sources, in their case stickslip tremor at the base of a glacier. In such a scenario, where clear identification of phase arrivals is difficult, our approach may be a more systematic approach and help give improved estimates of the source mechanism.

\subsection{Secondary Microseism}

In a second example, we locate seismic sources in the secondary microseism frequency band $(0.13$ to $0.15 \mathrm{~Hz}$ ) in the Northeastern Atlantic and Mediterrenean Sea using 342 stations distributed over Europe during the first week of February 2019 (Fig. 6). Three snapshots of beampower distributions are compared against hindcasts of significant wave height (WaveWatch III, Ardhuin et al., 2011). On first order, we find a good match between the standard approach ( $v=3.2 \mathrm{~km} / \mathrm{s}$, left), our approach (middle), and the distribution of significant wave height (right). A good match between seismic wave excitation and ocean wave activity is expected for the secondary microseism. The common explanation is that ocean gravity waves at the water surface, propagating in roughly opposite direction, interact and cause a standing wave that generates a vertically-propagating pressure wave 
non-peer reviewed preprint submitted to EarthArXiv

field in the water column. This pressure wave field then interacts with the ocean bottom, generating seismic waves in the solid Earth (Hasselmann, 1963; Ardhuin et al., 2015).

The similarity between the standard approach and our approach is high (Fig. 6 left and middle). This is not surprising for a number of reasons. The sources we image here are generally far away from most stations and towards one direction, West. The difference in waveforms recorded across all stations then becomes relatively small. If sources were closer to all stations, as e.g., for the Chino Hills earthquake (Fig. 5), improving the accuracy of the synthetic wave field has larger impact. Furthermore, the Green's function we rely on are based on an axisymmetric 1D Earth. Therefore we do not yet incorporate the full complexity of elastic wave propagation in this demonstration. Particularly relevant are likely the European shelf areas and the structure and velocity contrast between oceanic and continental crust. Finally, because we investigate the secondary microseism, we are limited to a narrow frequency band and cannot benefit from utilising broadband seismic waveforms, for which we believe our approach should perform a lot better than the standard approach. Still, we do find that beampower distributions retrieved with our approach are more focused on specific regions compared to the standard approach. We do not yet feel comfortable in judging whether these differences are certain to be an improvement in source estimation.

Our synthetic tests (Fig. 2) and the Chino Hills earthquake example (Fig. 5) suggests that our approach can be more precise in locating the sources. However, we have to be careful with interpreting these patterns, as we have also demonstrated in synthetic tests (Fig. 4). If our approach will prove to be more precise also for microseisms, we may find that seismic waves are excited in specific regions in the oceans and not distributed homogeneously beneath storm systems. It is important to note here that for now we use an explosion source mechanism for the synthetic wave fields to locate the microseism, which we have already shown to be inadequate for an earthquake (Fig. 5). In the future, we require a strategy to describe and incorporate a source mechanisms appropriate for microseisms. Some insight in how that could be approached has been given by Gualtieri et al. (2020) and this is certainly an attractive prospect and may help better understand the exact excitation mechanism.

\section{Conclusions}

Matched Field Processing (MFP) is generalized Beamforming for arbitrary wave fields, removing the need for the plane-wave assumption. It is one of the current approaches to locating sources of 
non-peer reviewed preprint submitted to EarthArXiv

ambient seismic noise (Fig. 1). In this study, we advance MFP to better incorporate elastic wave propagation in the Earth by using Green's functions numerically computed for real Earth structure directly as the synthetic wave field that the data is matched against.

When amplitudes are considered in MFP, results are biased by amplitude effects such as geometrical spreading and anelastic attenuation. In the standard approach, this is usually neglected through spectral whitening of the synthetic wave field. We find that this strategy performs best for us as well, and that trying to correct for spreading and attenuation via an amplitude term, as has been suggested before, may not be advisable (Fig. 3). This is especially the case for our approach, where multiple wave types can be considered simultaneously.

Two examples on real data showcase the potential of our approach (Figs. 5, 6). In principle, we can use it to search for the source mechanism of a seismic source, as suggested by the improved source location after incoporating the earthquake's moment tensor (Fig. 5). This could be particularly useful in the context of tremor activity, where source mechanism determination is challenging with classical approaches. In a second example, we locate sources of the secondary microseism in the Northern Atlantic and Mediterrenean Sea (Fig. 6). Results from our approach match the standard approach's results closely, likely due to source geometry, narrow frequency band, and our reliance on Green's functions computed for an axisymmetric Earth. Our approach retains the advantage that is not biased by author choice of a constant velocity, and potentially provides higher resolution.

We clarify conceptual approaches to MFP and its close connection to the interferometry-based localisation. The striking similarity between them suggests that it may be a worthwhile endeavour to unify them in the future, or at least provide a framework to let the different communities benefit from each others' work. MFP in particular would benefit tremendously from an approach for quantifying its resolution. The lack of such a measure is currently its major disadvantage.

Future advances focused on MFP for real Earth structure could be on more precise Green's functions databases, or investigating the performance of beamformers particularly for elastic wave propagation. With current tools, there is the potential for reasonably sized databases that incorporate true 3D Earth structure when limitting source locations to be only at the surface. More precise Green's functions should also incorporate a better description of the microseism source mechanism, different for the primary and secondary microseism. Our approach could improve MFP with few and sparsely distributed stations, because it is less reliant on waveform-coherency across seismic stations in its strict sense. While seismometer density is improving worldwide consistently, regions with sparse deployments and without purposefully built arrays are still the norm. Furthermore, tremor activity 
non-peer reviewed preprint submitted to EarthArXiv

such as volcanic tremor is often challenging to locate with classical approaches. Particularly in such regions and study targets, MFP is a powerful strategy for localising the origin of seismic energy.

\section{Data and Materials}

We provide all data and code used to generate the figures in this paper to make it entirely reproducible (https://github.com/seismology-hamburg/schippkus_hadziioannou_2022). There, we also provide a minimal working MFP example based on synthetic data and the standard approach to make the method more accessible for students and researchers interested in MFP. The MFP computations in this study rely on Python code developed for this work, which we make available under MIT license at https://github.com/seismology-hamburg/matched_field_processing.

Seismic data used in this study was provided by network operators of international, national, and regional seismic networks in Europe and America (California Institute of Technology and United States Geological Survey Pasadena, 1926; ZAMG - Zentralanstalt für Meterologie und Geodynamik, 1987; Institut fuer Geowissenschaften, Friedrich-Schiller-Universitaet Jena, 2009; University of Leipzig, 2001; Ruhr Universitaet Bochum (RUB Germany), 2007; RESIF, 2018; Instituto Português do Mar e da Atmosfera, I.P., 2006; Istituto Nazionale di Oceanografia e di Geofisica Sperimentale OGS, 2016; Norsar, 1971; KNMI, 1993; MedNet Project Partner Institutions, 1988; Instituto Dom Luiz (IDL) - Faculdade de Ciências da Universidade de Lisboa, 2003; San Fernando Royal Naval Observatory (ROA) et al., 1996; INGV Seismological Data Centre, 1997; Albuquerque Seismological Laboratory (ASL)/USGS, 1988; Scripps Institution of Oceanography, 1986; University of Genoa, 1967; Institut De Physique Du Globe De Paris (IPGP) \& Ecole Et Observatoire Des Sciences De La Terre De Strasbourg (EOST), 1982; GEOFON Data Centre, 1993; Dublin Institute for Advanced Studies, 1993; Charles University in Prague (Czech) et al., 1973; University of Zagreb, 2001; Swiss Seismological Service (SED) At ETH Zurich, 1983; Department of Earth and Environmental Sciences, Geophysical Observatory, University of Munchen, 2001; Royal Observatory of Belgium, 1985; Slovenian Environment Agency, 1990; RESIF, 1995; Federal Institute for Geosciences and Natural Resources, 1976) and accessed through ORFEUS, EIDA, and IRIS via obspy (Krischer et al., 2015). Colormaps used in this study are perceptually uniform (Crameri et al., 2020). 
non-peer reviewed preprint submitted to EarthArXiv

\section{Acknowledgements}

The authors thank Josefine Umlauft and Jonas Igel for insightful discussions of the ideas presented in this paper.

\section{References}

Aki, K., 1957. Space and time spectra of stationary stochastic waves, with special reference to microtremors, Bulletin of the Earthquake Research Institute, 35, 415-457.

Albuquerque Seismological Laboratory (ASL)/USGS, 1988. Global seismograph network IRIS/USGS.

Ardhuin, F., Stutzmann, E., Schimmel, M., \& Mangeney, A., 2011. Ocean wave sources of seismic noise, Journal of Geophysical Research, 116(C9), C09004-21.

Ardhuin, F., Gualtieri, L., \& Stutzmann, E., 2015. How ocean waves rock the Earth: Two mechanisms explain microseisms with periods 3 to $300 \mathrm{~s}, \mathrm{pp} .1-8$.

Baggeroer, A., Kuperman, W., \& Mikhalevsky, P., 1993. An overview of matched field methods in ocean acoustics, IEEE Journal of Oceanic Engineering, 18(4), 401-424.

Bensen, G. D., Ritzwoller, M. H., Barmin, M. P., Levshin, A. L., Lin, F., Moschetti, M. P., Shapiro, N. M., \& Yang, Y., 2007. Processing seismic ambient noise data to obtain reliable broad-band surface wave dispersion measurements, Geophysical Journal International, 169(3), 1239-1260.

Bowden, D. C., Sager, K., Fichtner, A., \& Chmiel, M., 2020. Connecting beamforming and kernelbased noise source inversion, Geophysical Journal International.

Brenguier, F., Campillo, M., Hadziioannou, C., Shapiro, N. M., Nadeau, R. M., \& Larose, E., 2008. Postseismic relaxation along the San Andreas fault at Parkfield from continuous seismological observations., Science (New York, N.Y.), 321(5895), 1478-1481.

Brenguier, F., Boué, P., Ben-Zion, Y., Vernon, F. L., Johnson, C. W., Mordret, A., Coutant, O., Share, P. E., Beaucé, E., Hollis, D., \& Lecocq, T., 2019. Train traffic as a powerful noise source for monitoring active faults with seismic interferometry, Geophysical Research Letters, 46(16), 9529-9536. 
non-peer reviewed preprint submitted to EarthArXiv

Brienzo, R. K. \& Hodgkiss, W. S., 1993. Broadband matched-field processing, The Journal of the Acoustical Society of America, 94(5), 2821-2831.

Bucker, H. P., 1976. Use of calculated sound fields and matched-field detection to locate sound sources in shallow water, The Journal of the Acoustical Society of America, 59(2), 368-373.

Burtin, A., Bollinger, L., Vergne, J., Cattin, R., \& Nábělek, J. L., 2008. Spectral analysis of seismic noise induced by rivers: A new tool to monitor spatiotemporal changes in stream hydrodynamics, Journal of Geophysical Research: Solid Earth, 113(B5).

California Institute of Technology and United States Geological Survey Pasadena, 1926. Southern california seismic network.

Campillo, M. \& Roux, P., 2015. Crust and lithospheric structure - seismic imaging and monitoring with ambient noise correlations, in Treatise on Geophysics, pp. 391-417, Elsevier, Oxford.

Capon, J., 1969. High-resolution frequency-wavenumber spectrum analysis, Proceedings of the IEEE, 57(8), 1408-1418.

Charles University in Prague (Czech), Institute of Geonics, Institute of Geophysics, Academy of Sciences of the Czech Republic, Institute of Physics of the Earth Masaryk University (Czech), \& Institute of Rock Structure and Mechanics, 1973. Czech regional seismic network.

Corciulo, M., Roux, P., Campillo, M., Dubucq, D., \& Kuperman, W. A., 2012. Multiscale matchedfield processing for noise-source localization in exploration geophysics, GEOPHYSICS, 77(5), KS33-KS41.

Cox, H., 2000. Multi-rate adaptive beamforming (MRABF), in Proceedings of the IEEE Sensor Array and Multichannel Signal Processing Workshop, pp. 306-309, ORINCON Corporation, Arlington, United States.

Cox, H., Zeskind, R., \& Owen, M., 1987. Robust adaptive beamforming, IEEE Transactions on Acoustics, Speech, and Signal Processing, 35(10), 1365-1376.

Crameri, F., Shephard, G. E., \& Heron, P. J., 2020. The misuse of colour in science communication, Nature Communications, pp. 1-10.

Dean, T., 2019. The seismic signature of rain, ASEG Extended Abstracts, 2018(1), 1-8. 
non-peer reviewed preprint submitted to EarthArXiv

DeMuth, G., 1977. Frequency domain beamforming techniques, in ICASSP '77. IEEE International Conference on Acoustics, Speech, and Signal Processing, vol. 2, pp. 713-715.

Department of Earth and Environmental Sciences, Geophysical Observatory, University of Munchen, 2001. BayernNetz.

D'Spain, G. L., Murray, J. J., Hodgkiss, W. S., Booth, N. O., \& Schey, P. W., 1999. Mirages in shallow water matched field processing, The Journal of the Acoustical Society of America, 105(6), 3245-3265.

Dublin Institute for Advanced Studies, 1993. Irish national seismic network.

Dziewonski, A. M. \& Anderson, D. L., 1981. Preliminary reference Earth model, Physics of the Earth and Planetary Interiors, 25(4), 297-356.

Ermert, L., Villaseñor, A., \& Fichtner, A., 2016. Cross-correlation imaging of ambient noise sources, Geophysical Journal International, 204(1), 347-364.

Ermert, L., Sager, K., Nissen-Meyer, T., \& Fichtner, A., 2021. Multi-frequency inversion of global ambient seismic sources, Geophysical Journal International.

Federal Institute for Geosciences and Natural Resources, 1976. German regional seismic network (GRSN).

Fichtner, A., Stehly, L., Ermert, L., \& Boehm, C., 2017. Generalized interferometry - I: Theory for interstation correlations, Geophysical Journal International, 208(2), 603-638.

Fichtner, A., Bowden, D., \& Ermert, L., 2020. Optimal processing for seismic noise correlations, Geophysical Journal International, 223(3), 1548-1564.

Fuchs, F., Bokelmann, G. H. R., \& the AlpArray Working Group, 2017. Equidistant spectral lines in train vibrations, Seismological Research Letters, 89(1), 56-66.

Gal, M. \& Reading, A. M., 2019. Beamforming and polarisation analysis, Seismic Ambient Noise, pp. 32-72.

Gal, M., Reading, A. M., Ellingsen, S. P., Koper, K. D., Gibbons, S. J., \& Näsholm, S. P., 2014. Improved implementation of the $\mathrm{fk}$ and Capon methods for array analysis of seismic noise, Geophysical Journal International, 198(2), 1045-1054. 
non-peer reviewed preprint submitted to EarthArXiv

Gal, M., Reading, A. M., Rawlinson, N., \& Schulte-Pelkum, V., 2018. Matched field processing of three-component seismic array data applied to rayleigh and love microseisms, Journal of Geophysical Research: Solid Earth, 42(4), 765-19.

GEOFON Data Centre, 1993. GEOFON seismic network.

Gibbons, S. J., Harris, D. B., Kvaerna, T., \& Dodge, D. A., 2017. Comprehensive Seismic Detection and Estimation Using Matched Field Processing, Technical Report AD1056994, Air Force Resarch Laboratory, Kirtland Air Force Base, NM.

Gradon, C., Moreau, L., Roux, P., \& Ben-Zion, Y., 2019. Analysis of surface and seismic sources in dense array data with match field processing and Markov chain Monte Carlo sampling, Geophysical Journal International, 218(2), 1044-1056.

Gualtieri, L., Bachmann, E., Simons, F. J., \& Tromp, J., 2020. The origin of secondary microseism Love waves., Proceedings of the National Academy of Sciences of the United States of America, 117(47), 29504-29511.

Hadziioannou, C., Larose, E., Baig, A., Roux, P., \& Campillo, M., 2011. Improving temporal resolution in ambient noise monitoring of seismic wave speed, Journal of Geophysical Research, 116(B7), B08310-10.

Hasselmann, K., 1963. A statistical analysis of the generation of microseisms, Reviews of Geophysics, 1(2), 177-210.

Heimann, S., Kriegerowski, M., Isken, M., Cesca, S., Daout, S., Grigoli, F., Juretzek, C., Megies, T., Nooshiri, N., Steinberg, A., Sudhaus, H., Vasyura-Bathke, H., Willey, T., \& Dahm, T., 2017. Pyrocko - An open-source seismology toolbox and library.

Hu, W., Barthelmie, R. J., Letson, F., \& Pryor, S. C., 2019. Seismic noise induced by wind turbine operation and wind gusts, Seismological Research Letters, 91(1), 427-437.

Hutko, A. R., Bahavar, M., Trabant, C., Weekly, R. T., Fossen, M. V., \& Ahern, T., 2017. Data Products at the IRIS-DMC: Growth and Usage, Seismological Research Letters, 88(3), 892-903.

Igel, J., Bowden, D., Sager, K., \& Fichtner, A., 2021a. Imaging noise sources: Comparing and combining a matched-field processing technique with finite-frequency noise source inversions, in EGU General Assembly 2021, online. 
non-peer reviewed preprint submitted to EarthArXiv

Igel, J. K. H., Ermert, L. A., \& Fichtner, A., 2021b. Rapid finite-frequency microseismic noise source inversion at regional to global scales, Geophysical Journal International, (ggab210).

INGV Seismological Data Centre, 1997. Rete sismica nazionale (RSN).

Institut De Physique Du Globe De Paris (IPGP) \& Ecole Et Observatoire Des Sciences De La Terre De Strasbourg (EOST), 1982. GEOSCOPE, French Global Network of broad band seismic stations.

Institut fuer Geowissenschaften, Friedrich-Schiller-Universitaet Jena, 2009. Thüringer seismologisches netz.

Instituto Dom Luiz (IDL) - Faculdade de Ciências da Universidade de Lisboa, 2003. University of lisbon seismic network.

Instituto Português do Mar e da Atmosfera, I.P., 2006. Portuguese national seismic network.

Istituto Nazionale di Oceanografia e di Geofisica Sperimentale - OGS, 2016. North-east italy seismic network.

Johnson, C. W., Meng, H., Vernon, F. L., \& Ben-Zion, Y., 2019. Characteristics of ground motion generated by wind interaction with trees, structures, and other surface obstacles, Journal of Geophysical Research: Solid Earth, 124(8), 8519-8539.

Juretzek, C. \& Hadziioannou, C., 2016. Where do ocean microseisms come from? A study of Love-to-Rayleigh wave ratios, Journal of Geophysical Research: Solid Earth, 121(9), 6741-6756.

Juretzek, C. \& Hadziioannou, C., 2017. Linking source region and ocean wave parameters with the observed primary microseismic noise, Geophysical Journal International, 211(3), 1640-1654.

KNMI, 1993. Netherlands seismic and acoustic network.

Krim, H. \& Viberg, M., 1996. Two decades of array signal processing research: The parametric approach, IEEE Signal Processing Magazine, 13(4), 67-94.

Krischer, L., Megies, T., Barsch, R., Beyreuther, M., Lecocq, T., Caudron, C., \& Wassermann, J., 2015. ObsPy: A bridge for seismology into the scientific Python ecosystem, Computational Science \& Discovery, 8(014003). 
non-peer reviewed preprint submitted to EarthArXiv

Krischer, L., Hutko, A. R., van Driel, M., Stähler, S., Bahavar, M., Trabant, C., \& Nissen-Meyer, T., 2017. On-Demand Custom Broadband Synthetic Seismograms, Seismological Research Letters, 88(4), 1127-1140.

Li, F., Zhu, F., Zhang, Y., Zhang, B., Li, W., \& Luo, W., 2021. Synthetic adaptive matched field processing for moving source with a horizontal line array, The Journal of the Acoustical Society of America, 149(2), 1138-1146.

Liu, Y., Yue, Y., Luo, Y., \& Li, Y., 2021. Effects of high-speed train traffic characteristics on seismic interferometry, Geophysical Journal International, 227(1), 16-32.

Löer, K., Riahi, N., \& Saenger, E. H., 2018. Three-component ambient noise beamforming in the Parkfield area, Geophysical Journal International, 213(3), 1478-1491.

Longuet-Higgings, M. S., 1950. A theory of the origin of microseisms, Philosophical Transactions of the Royal Society of London A Mathematical, Physical and Engineering Sciences, 243(857), $1-35$

Lu, Y., Stehly, L., Paul, A., \& the AlpArray Working Group, 2018. High-resolution surface wave tomography of the European crust and uppermost mantle from ambient seismic noise, Geophysical Journal International, 214(2), 1136-1150.

MedNet Project Partner Institutions, 1988. Mediterranean very broadband seismographic network (MedNet).

eds Nakata, N., Gualtieri, L., \& Fichtner, A., 2019. Seismic Ambient Noise, Cambridge University Press, Cambridge.

Nissen-Meyer, T., van Driel, M., Stähler, S. C., Hosseini, K., Hempel, S., Auer, L., Colombi, A., \& Fournier, A., 2014. AxiSEM: Broadband 3-D seismic wavefields in axisymmetric media, Solid Earth, 5(1), 425-445.

Norsar, 1971. NORSAR station network.

Paul, A., Campillo, M., Margerin, L., Larose, E., \& Derode, A., 2005. Empirical synthesis of time-asymmetrical Green functions from the correlation of coda waves, Journal of Geophysical Research, 110(B8), B08302-13. 
non-peer reviewed preprint submitted to EarthArXiv

RESIF, 1995. RESIF-RLBP French Broad-band network, RESIF-RAP strong motion network and other seismic stations in metropolitan France.

RESIF, 2018. CEA/DASE broad-band permanent network in metropolitan France.

Riahi, N. \& Gerstoft, P., 2015. The seismic traffic footprint: Tracking trains, aircraft, and cars seismically, Geophysical Research Letters, 42(8), 2674-2681.

Riahi, N., Bokelmann, G., Sala, P., \& Saenger, E. H., 2013. Time-lapse analysis of ambient surface wave anisotropy: A three-component array study above an underground gas storage: SURFACE WAVE NOISE ANISOTROPY OVER TIME, Journal of Geophysical Research: Solid Earth, 118(10), 5339-5351.

Rost, S. \& Thomas, C., 2002. Array Seismology: Methods and Applications, Reviews of Geophysics, 40(3), 2-1-2-27.

Royal Observatory of Belgium, 1985. Belgian seismic network.

Ruhr Universitaet Bochum (RUB Germany), 2007. RuhrNet - seismic network of the ruhr-university bochum.

Ruigrok, E., Gibbons, S., \& Wapenaar, K., 2017. Cross-correlation beamforming, Journal of Seismology, 21(3), 495-508.

Sager, K., Boehm, C., Ermert, L., Krischer, L., \& Fichtner, A., 2018. Sensitivity of Seismic Noise Correlation Functions to Global Noise Sources, Journal of Geophysical Research: Solid Earth, 123(8), 6911-6921.

San Fernando Royal Naval Observatory (ROA), Universidad Complutense De Madrid (UCM), Helmholtz-Zentrum Potsdam Deutsches GeoForschungsZentrum (GFZ), Universidade De Evora (UEVORA, Portugal), \& Institute Scientifique Of RABAT (ISRABAT, Morocco), 1996. The western mediterranean BB seismic network.

SCEDC, 2013. Southern California Earthquake Center.

Schimmel, M. \& Gallart, J., 2003. The use of instantaneous polarization attributes for seismic signal detection and image enhancement, Geophysical Journal International, 155(2), 653-668. 
non-peer reviewed preprint submitted to EarthArXiv

Schimmel, M., Stutzmann, E., Ardhuin, F., \& Gallart, J., 2011. Polarized Earth's ambient microseismic noise, Geochemistry, Geophysics, Geosystems, 12(7).

Schippkus, S., Zigone, D., Bokelmann, G. H. R., \& the AlpArray Working Group, 2018. Ambientnoise tomography of the wider Vienna Basin region, Geophysical Journal International, 215(1), $102-117$.

Schippkus, S., Garden, M., \& Bokelmann, G. H. R., 2020. Characteristics of the ambient seismic field on a large-n seismic array in the vienna basin, Seismological Research Letters.

Schmidt, R., 1986. Multiple emitter location and signal parameter estimation, IEEE Transactions on Antennas and Propagation, 34(3), 276-280.

Scripps Institution of Oceanography, 1986. Global seismograph network - IRIS/IDA.

Sergeant, A., Chmiel, M., Lindner, F., Walter, F., Roux, P., Chaput, J., Gimbert, F., \& Mordret, A., 2020. On the Green's function emergence from interferometry of seismic wave fields generated in high-melt glaciers: Implications for passive imaging and monitoring, The Cryosphere, 14(3), $1139-1171$.

Sethian, J. A., 1999. Fast Marching Methods, SIAM Review, 41(2), 199-235.

Shapiro, N. M., Campillo, M., Stehly, L., \& Ritzwoller, M. H., 2005. High-resolution surface-wave tomography from ambient seismic noise., Science, 307(5715), 1615-1618.

Slovenian Environment Agency, 1990. Seismic network of the republic of slovenia.

Smith, K. \& Tape, C., 2019. Seismic Noise in Central Alaska and Influences From Rivers, Wind, and Sedimentary Basins, Journal of Geophysical Research: Solid Earth, 124(11), 11678-11704.

Soares, C. \& Jesus, S. M., 2003. Broadband matched-field processing: Coherent and incoherent approaches, The Journal of the Acoustical Society of America, 113(5), 2587-2598.

Stammler, K. \& Ceranna, L., 2016. Influence of wind turbines on seismic records of the gräfenberg array, Seismological Research Letters, 87(5), 1075-1081.

Swiss Seismological Service (SED) At ETH Zurich, 1983. National seismic networks of switzerland. 
non-peer reviewed preprint submitted to EarthArXiv

Tolstoy, A., 1989. Sensitivity of matched field processing to sound-speed profile mismatch for vertical arrays in a deep water Pacific environment, The Journal of the Acoustical Society of America, 85(6), 2394-2404.

Umlauft, J. \& Korn, M., 2019. 3-D fluid channel location from noise tremors using matched field processing, Geophysical Journal International, 219(3), 1550-1561.

Umlauft, J., Lindner, F., Roux, P., Mikesell, T. D., Haney, M. M., Korn, M., \& Walter, F. T., 2021. Stick-Slip Tremor Beneath an Alpine Glacier, Geophysical Research Letters, 48(2).

University of Genoa, 1967. Regional seismic network of north western italy.

University of Leipzig, 2001. SXNET saxon seismic network.

University of Zagreb, 2001. Croatian seismograph network.

van Driel, M., Krischer, L., Stähler, S. C., Hosseini, K., \& Nissen-Meyer, T., 2015. Instaseis: Instant global seismograms based on a broadband waveform database, Solid Earth, 6(2), 701-717.

Wapenaar, K., Draganov, D., Snieder, R., Campman, X., \& Verdel, A., 2010. Tutorial on seismic interferometry: Part 1 - Basic principles and applications, GEOPHYSICS, 75(5), 75A19575A209.

Yong, P., Liao, W., Huang, J., Li, Z., \& Lin, Y., 2019. Misfit function for full waveform inversion based on the Wasserstein metric with dynamic formulation, Journal of Computational Physics, 399, 108911.

ZAMG - Zentralanstalt für Meterologie und Geodynamik, 1987. Austrian seismic network.

Zhu, G., Wang, Y., \& Wang, Q., 2020. Matched Field Processing Based on Bayesian Estimation, Sensors, 20(5), 1374.

Ziane, D. \& Hadziioannou, C., 2019. The contribution of multiple scattering to Love wave generation in the secondary microseism, Geophysical Journal International, 217(2), 1108-1122. 
non-peer reviewed preprint submitted to EarthArXiv

\section{Figures}

a) Polarization Analysis

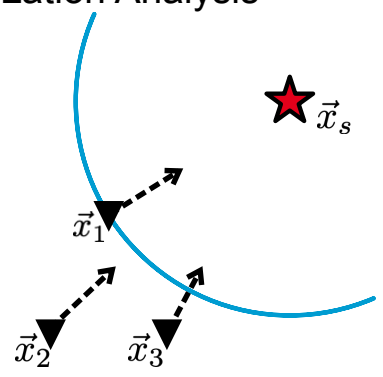

c) Interferometry-based

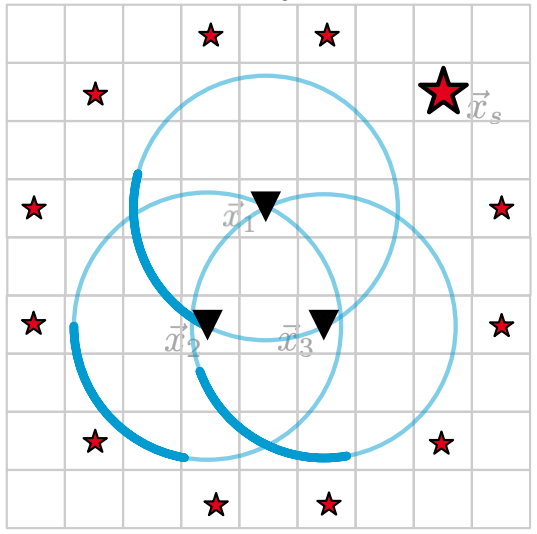

b) Beamforming

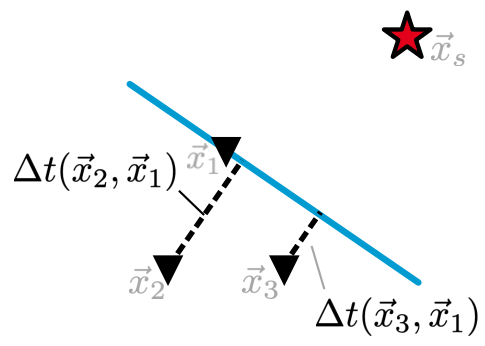

d) Matched Field Processing

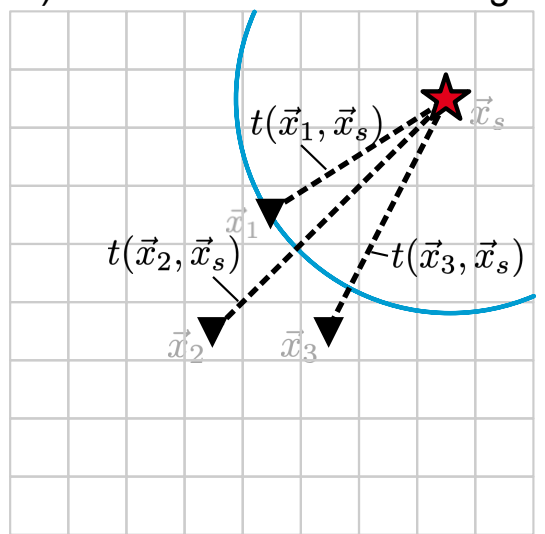

Figure 1: Current approaches to locating sources of the ambient seismic field. Wavefronts are marked blue. a) Polarization Analysis: The polarization of the wave field on individual threecomponent seismometers gives an indication of direction of propagation. Triangulation allows source localisation. b) Beamforming: Seismograms on multiple stations are shifted in time corresponding to candidate plane-waves, and summed over. c) Interferometry-based strategy: Compare crosscorrelation functions computed from seismograms of multiple stations with synthetically computed cross-correlation functions for a given source distribution. Cross-correlation functions are sensitive to the source distribution and are asymmetric (indicated by thickness of wavefront), if sources are distributed heterogeneously. d) Matched Field Processing is generalized Beamforming, sampling candidate source locations. Allows for curved wavefronts. 
non-peer reviewed preprint submitted to EarthArXiv
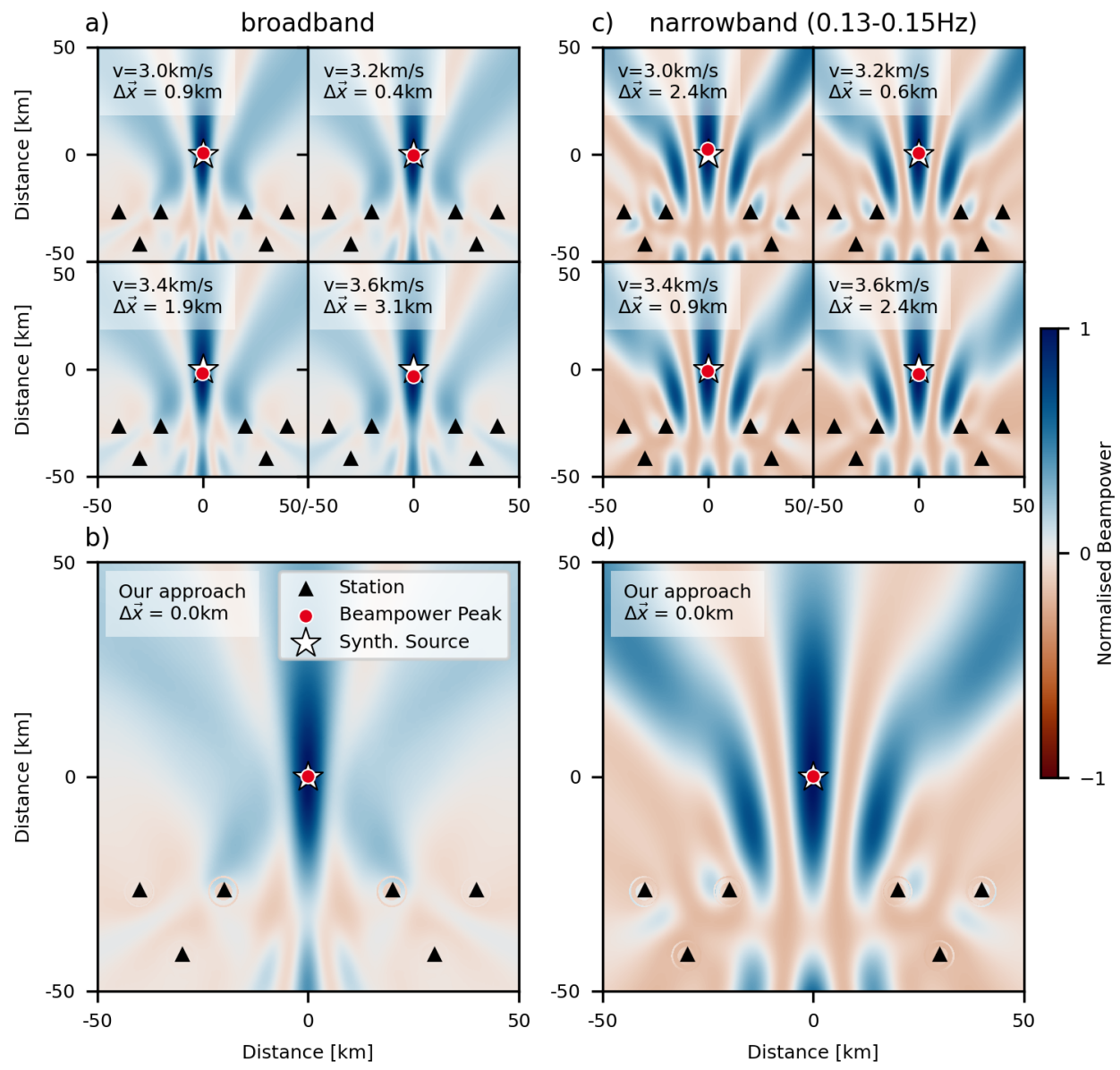

Figure 2: Synthetic demonstration for two three-station arrays locating an explosion source. The grid point with the highest beampower is the estimated source location (red circle). Left: broadband source. a) Standard approach, with travel times estimated for constant velocity. The retrieved source location is sensitive to the chosen velocity. b) Our approach, with numerical Green's functions as synthetic wave fields. Source is precisely located. Right: narrowband source $(0.13-0.15 \mathrm{~Hz})$. c) Standard approach. Emergence of sidelobes due to interference. d) Our approach in the same narrow frequency band. Source is precisely located. 
non-peer reviewed preprint submitted to EarthArXiv

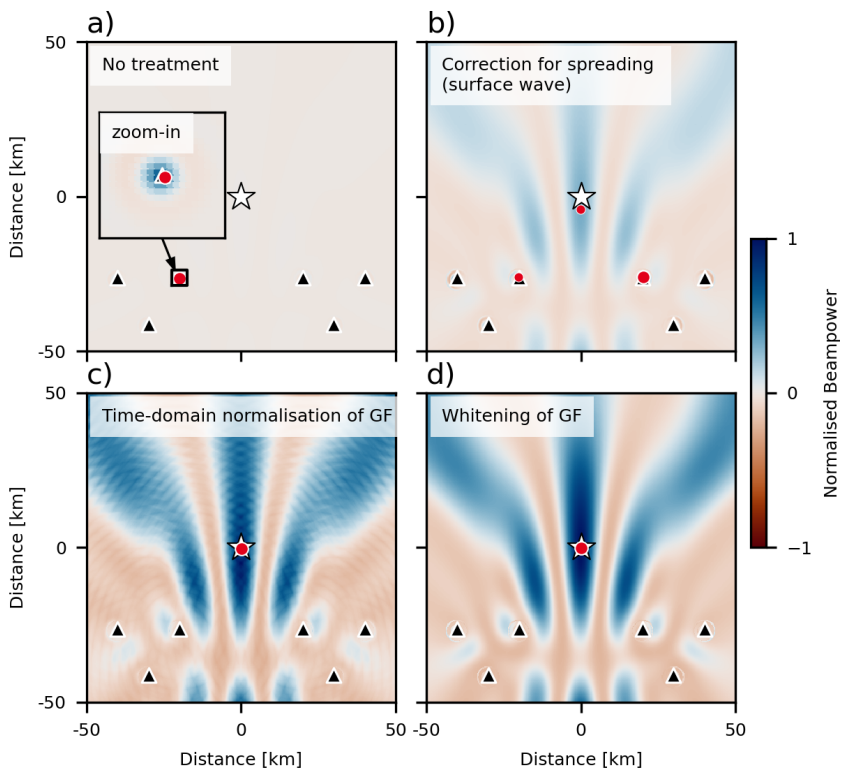

Figure 3: Strategies for treating amplitude information. a) No amplitude treatment. b) Correction for geometrical spreading of surface waves. Smaller red circles mark local beampower maxima. c) Time-domain normalisation of numerical Green's function (GF). d) Spectral whitening (frequencydomain normalisation) of numerical Green's function (GF).
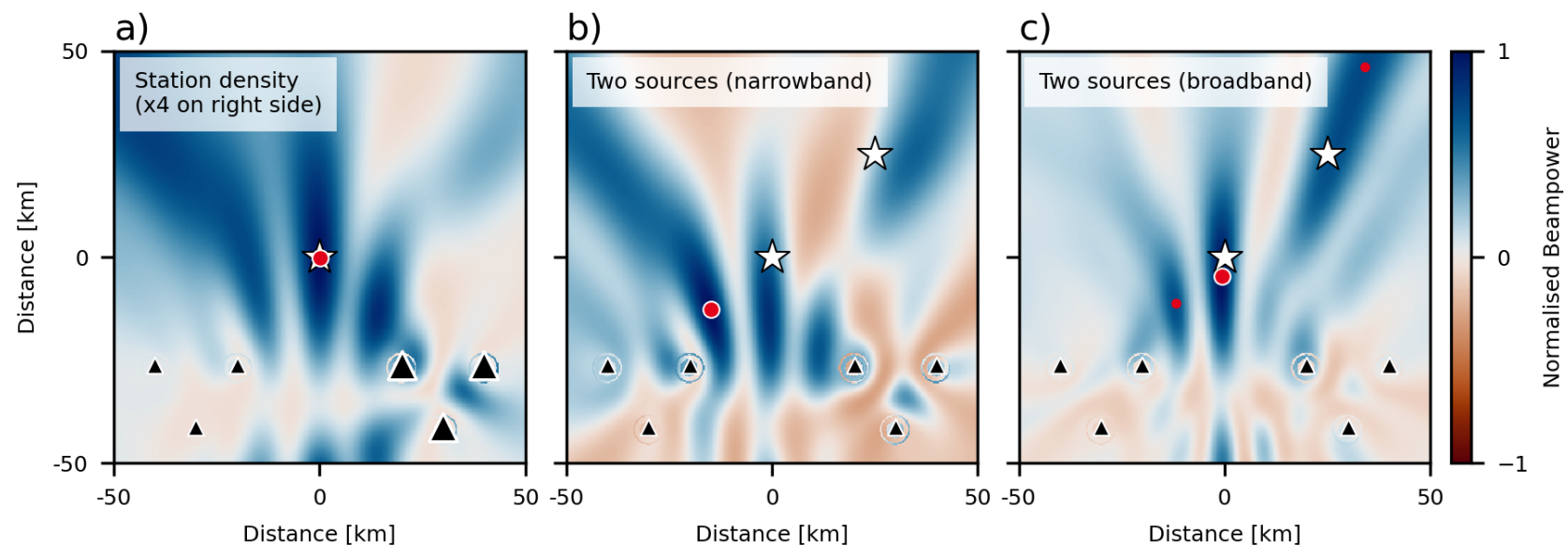

Figure 4: Some limitations of MFP, regardless of Green's function formulation. a) Impact of station density. Placing four times as many stations on the right side (indicated by larger triangles) results in visual bias of potential source locations. True source location is still resolved. b) Two narrowband (0.13 to $0.15 \mathrm{~Hz})$ sources active at the same time. Beampower distribution does not represent source locations well. Global beampower maximum is an interference artefact. c) Same as b), but for a broadband source. Smaller red circles mark local beampower maxima. Closer to real source locations, but still not well-resolved. 
non-peer reviewed preprint submitted to EarthArXiv

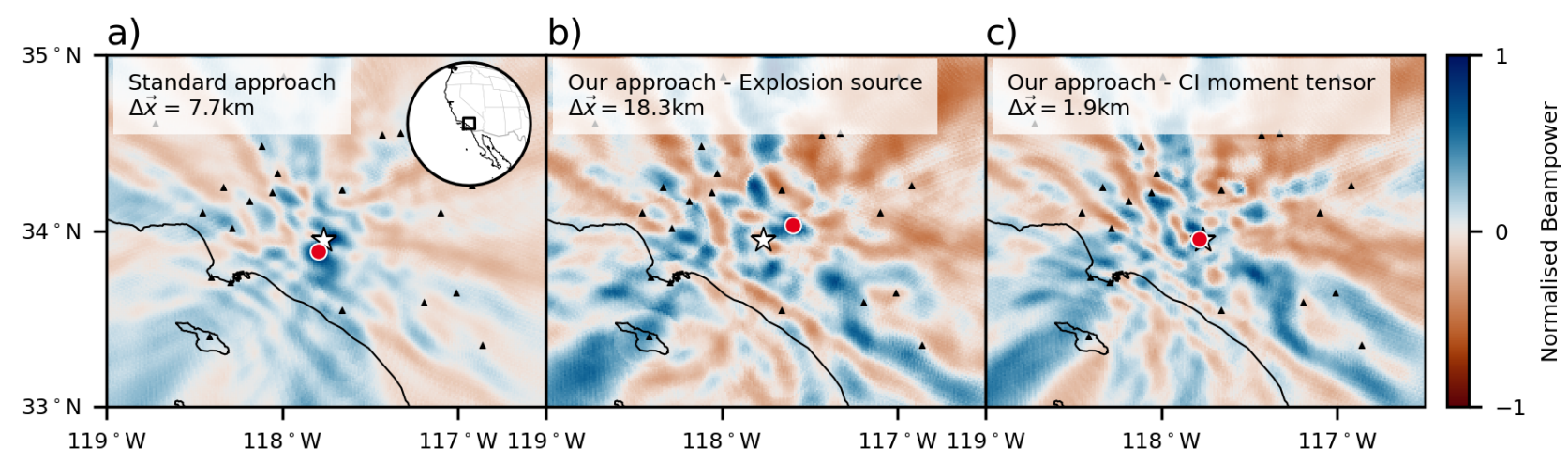

Figure 5: Location of the 2008-07-29 Chino Hills earthquake from the Cl catalog (white star, SCEDC, 2013) and MFP (red circle) at $15.5 \mathrm{~km}$ depth. MFP results were obtained using stations of the Southern California Seismic Network (black triangles) and frequencies from 0.1 to $0.2 \mathrm{~Hz}$. a) Beampower distribution with simple analytical Green's functions, assuming $v=3.2 \mathrm{~km} / \mathrm{s} .7 .7$ $\mathrm{km}$ distance to the $\mathrm{Cl}$ location. b) Beampower distribution using numerical Green's functions for an explosive source mechanism. $18.3 \mathrm{~km}$ distance to the $\mathrm{Cl}$ location. c) Beampower distribution using numerical Green's functions for the moment tensor solution in the $\mathrm{Cl}$ catalog (SCEDC, 2013). 1.9 $\mathrm{km}$ distance to the $\mathrm{Cl}$ location. 


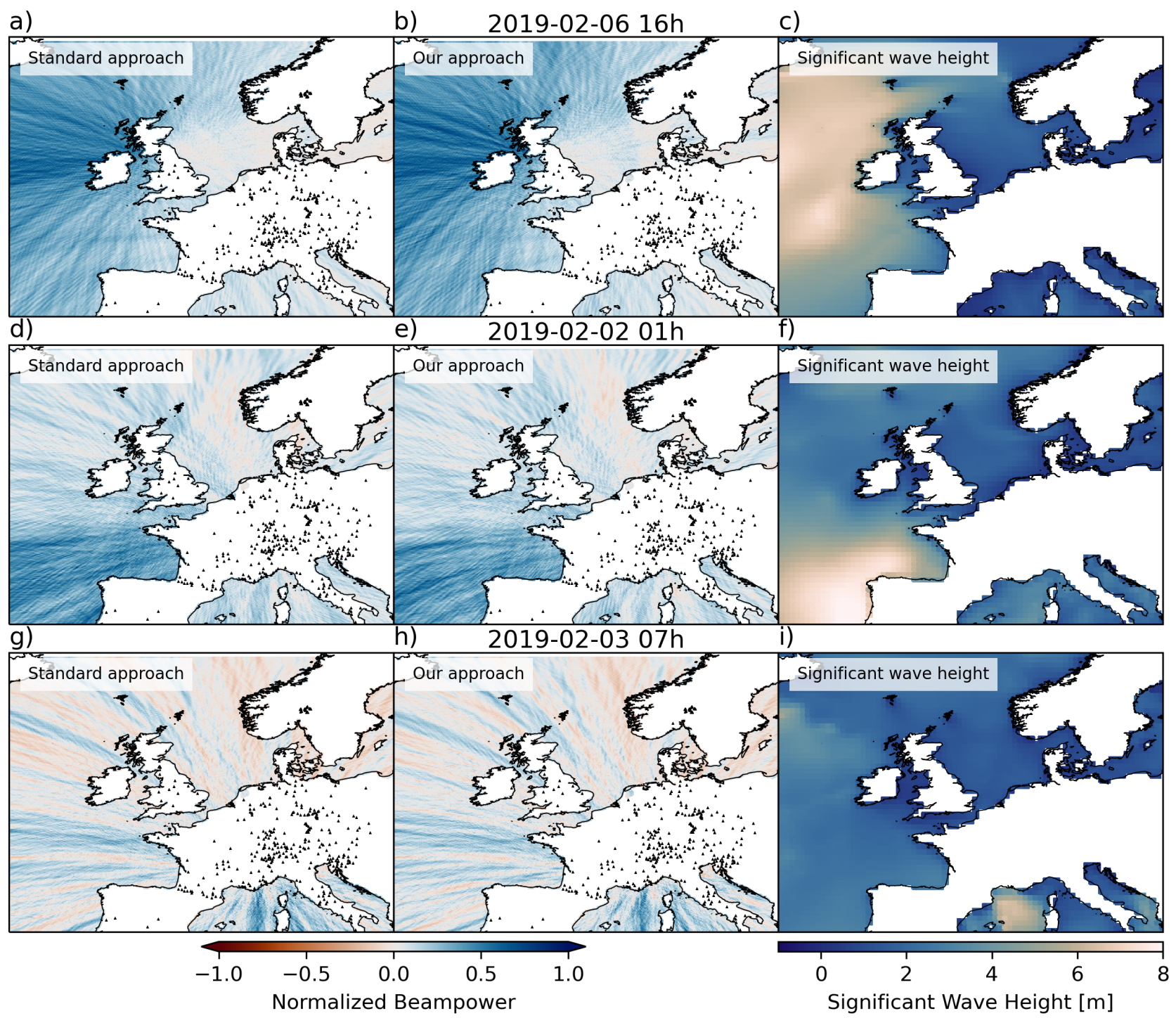

Figure 6: MFP results for the secondary microseism $(0.13$ to $0.15 \mathrm{~Hz})$ during the first week of February 2019 for three time windows (rows). 342 stations distributed over Europe were used (black triangles). Left: MFP using analytical Green's functions, assuming $v=3.2 \mathrm{~km} / \mathrm{s}$. Middle: MFP using numerically computed Green's functions (our approach). Right: Maps of significant wave height hindcasts, provided by WaveWatch III (Ardhuin et al., 2011). 